\title{
Debugging through Evaluation Sequences: A Controlled Experimental Study *
}

\author{
Zhenyu Zhang, Bo Jiang \\ The University of Hong Kong \\ Pokfulam, Hong Kong \\ $\{$ zyzhang,bjiang\}@cs.hku.hk
}

\author{
W. K. Chan \\ City University of Hong Kong \\ Tat Chee Avenue, Hong Kong \\ wkchan@cs.cityu.edu.hk
}

\author{
T. H. Tse ${ }^{\dagger}$ \\ The University of Hong Kong \\ Pokfulam, Hong Kong \\ thtse@cs.hku.hk
}

\begin{abstract}
Predicate-based statistical fault-localization techniques locate fault-relevant predicates in a program by contrasting the statistics of the values of individual predicates between successful and failure-causing runs. While short-circuit evaluations are common in program execution, treating predicates as atomic units ignores this fact, masking out various types of important statistics. On the contrary, are such statistics useful for debugging? In this paper, we investigate experimentally the impact of the use of short-circuit evaluation information on fault localization. The results show that, by doing so, it significantly improves predicate-based statistical fault-localization techniques.
\end{abstract}

Keywords: evaluation sequence, fault localization.

\section{Introduction}

Software debugging is a key activity in software development, and takes up a significant amount of resources in a typical project. Among the three major tasks of software debugging (namely, fault localization, fault repair, and regression testing of repaired programs), fault localization has been recognized as the hardest, tedious, and time-consuming [14]. Using an effective fault-localization technique to improve the productivity of programmers is a long-standing trend to alleviate the problem.

Recently, effective statistical fault-localization techniques were proposed. A strategy $[10,11]$ is to identify fault-relevant predicates rather than directly

* This project is supported in part by the General Research Fund of the Hong Kong Research Grants Council (projects no. 111107 and 716507).

$\dagger$ All correspondence should be addressed to Prof. T. H. Tse at Department of Computer Science, The University of Hong Kong, Pokfulam, Hong Kong. Tel: (+852) 2859 2183. Fax: (+852) 2858 4141. Email: thtse@cs.hku.hk. pinpointing the fault locations. This strategy holds the promise to sample a program in a lightweight manner to collect execution statistics, which also reduces the need to disclose the execution details of all statements when remote sampling is conducted (for the purpose of remote support rather than on-site support). Hence, it lowers the risk of information leakage, which is a security concern.

These techniques, however, require summarizing the execution statistics on individual predicates. A compound predicate may be executed in one way or another due to short-circuit evaluations over different sub-terms of the predicate. The execution statistics of a predicate is, therefore, the summary of a collection of lower-tier evaluations over different sub-terms. Is isolating these lower-tier evaluations beneficial in improving the effectiveness of predicate-based statistical fault-localization techniques? This paper conducts a controlled experimental investigation on the impact of the use of short-circuit evaluation sequences to improve statistical fault localization techniques.

We first give a few preliminaries. A successful test case is a test case showing no failures, and a failure-causing test case is one that detects a failure. A typical program contains numerous predicates in ifand while-statements. They are in the form of Boolean expressions, such as “ ${ }^{\prime} j<=1|| \operatorname{src}\left[{ }^{*} i+1\right]==^{\prime} \backslash 0^{\prime} \quad "$, which may comprise further conditions, such as “*j<=1" and " $\operatorname{src}\left[{ }^{*} i+1\right]==\prime \backslash 0^{\prime} "$ ".

Previous studies on statistical fault localization [10, 11] find the fault-relevant predicates in a program by counting the number of times $\left(n_{t}\right)$ a predicate is evaluated to be true in an execution as well as the number of times $\left(n_{f}\right)$ it is evaluated to be false, and then comparing these counts in various ways. The evaluation bias $\frac{n_{t}}{n_{t}+n_{f}}$ of a predicate is the percentage that it is evaluated to be true among all evaluations in a run [11].

The SOBER approach [11] proposes to contrast the differences between a set of evaluation biases due to successful test cases and that due to failure-causing ones 
for every predicate in the program. It hypothesizes that, the greater is the difference between such a pair of sets of evaluation biases, the higher will be the chance that the corresponding predicate is fault-relevant. The CBI approach [10] proposes a heuristic that measures the increase in probability that a predicate is evaluated to be true in a set of failure-causing test cases, compared to the whole set of (successful and failure-causing) test cases. These proposals are particularly interested in the evaluation results of predicates. They use the resultant values of the predicates to determine the counts.

A predicate can be considered as a Boolean expression. As mentioned above and to be discussed in Section 2, the resultant values of a Boolean expression may be due to different evaluation sequences. If we ignore the information on evaluation sequences, we may be masking out very useful statistics for effective fault localization. In this paper, we investigate whether the effect of a lower-tier concept - evaluation sequences - of predicates is significant on the effectiveness of predicate-based statistical fault localization. We set up a controlled experiment to study this question.

The major contributions of this paper are twofold: (i) We provide the first set of experimental results regarding the effect of short-circuit evaluations on statistical debugging. (ii) We show that short-circuit evaluation has a significant impact on the effectiveness of predicate-based fault-localization techniques. Indeed, the experimental result shows that the use of evaluation sequences can significantly improve on existing predicate-based statistical faultlocalization techniques.

We shall illustrate the potential of using evaluation sequences for fine-grained statistical fault localization in Section 2, which casts a scene for us to formulate the research questions in Section 3, followed by the associated experiment in Section 4. We shall next review related work in Section 5. Section 6 concludes the paper.

\section{A Motivating Study}

This section shows a motivating study we have conducted. It enables readers to have a feel of how the distribution of evaluation biases at the evaluation sequence level can be used to pinpoint a faulty predicate.

The upper part of Figure 1 shows a code fragment excerpted from the original version (version v0) of print_tokens2 from the Siemens suite of programs [5]. We have labeled the three individual conditions as $C_{1}$, $C_{2}$, and $C_{3}$, respectively. The lower part of the same

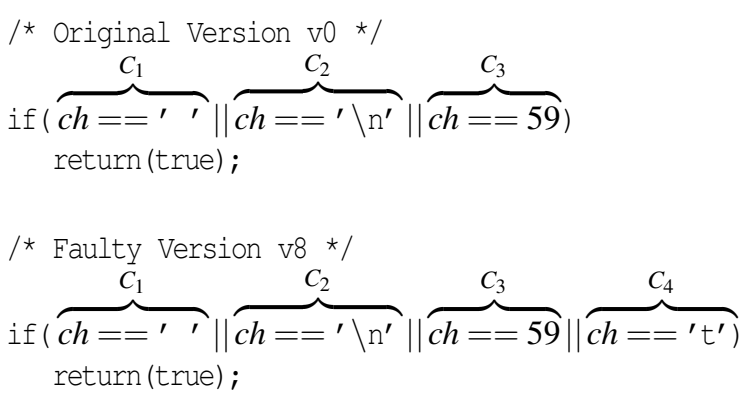

Figure 1. Code excerpts from versions v0 and v8 of print_tokens.

\begin{tabular}{|c|c|c|c|c||c|c|c|}
\hline $\mathrm{ES}$ & $C_{1}$ & $C_{2}$ & $C_{3}$ & $C_{4}$ & $\mathrm{v} 0$ & $\mathrm{v} 8$ & $\mathrm{v} 0=\mathrm{v} 8 ?$ \\
\hline \hline$e s_{1}$ & $\mathrm{~T}$ & $\perp$ & $\perp$ & $\perp$ & $\mathrm{T}$ & $\mathrm{T}$ & yes \\
\hline$e s_{2}$ & $\mathrm{~F}$ & $\mathrm{~T}$ & $\perp$ & $\perp$ & $\mathrm{T}$ & $\mathrm{T}$ & yes \\
\hline$e s_{3}$ & $\mathrm{~F}$ & $\mathrm{~F}$ & $\mathrm{~T}$ & $\perp$ & $\mathrm{T}$ & $\mathrm{T}$ & yes \\
\hline$e s_{4}$ & $\mathrm{~F}$ & $\mathrm{~F}$ & $\mathrm{~F}$ & $\mathrm{~T}$ & \multirow{2}{*}{$\mathrm{F}$} & $\mathrm{T}$ & no \\
\cline { 1 - 3 }$e s_{5}$ & $\mathrm{~F}$ & $\mathrm{~F}$ & $\mathrm{~F}$ & $\mathrm{~F}$ & & $\mathrm{~F}$ & yes \\
\hline
\end{tabular}

Table 1. Evaluation sequences of code fragments.

figure shows the code fragment excerpted from a faulty version (version v8) of the Siemens suite, where a fault was seeded into the predicate by adding an extra condition $\mathrm{ch}==^{\prime} \backslash \mathrm{t}^{\prime}$. We have labeled this condition as $C_{4}$.

Because of the effect of short-circuit rules of the $\mathrm{C}$ programming language on Boolean expressions, a condition in a Boolean expression may be evaluated to be true $(\mathrm{T})$ or false $(\mathrm{F})$, or may not be evaluated at all $(\perp)$. Furthermore, in terms of evaluations, the conditions on a Boolean expression can be seen as an ordered sequence. ${ }^{1}$ When a preceding condition in an evaluation sequence is not evaluated, by the shortcircuit rule, no succeeding condition in the evaluation sequence will be evaluated.

For the faulty Boolean expression in the fragment shown in Figure 1, there are five legitimate evaluation sequences $\left(e s_{1}\right.$ to $\left.e s_{5}\right)$, as shown in Table 1 . The columns under the individual conditions $\left(C_{1}\right.$ to $\left.C_{4}\right)$ represent the evaluation outcomes of the respective conditions based on the short-circuit rules of the programming language. In the column entitled v0, it shows the respective resultant values of the predicate in the original version of the program. In this column, the last two grids are merged because the two evaluation sequences ( $e s_{4}$ and $e s_{5}$ ) make no difference in the original program. The column entitled $\mathrm{v} 8$ shows the

\footnotetext{
${ }^{1}$ We simply consider every condition to be a distinct occurrence. In other words, even if two conditions in a predicate are identical, we consider them as two distinct occurrences.
} 
respective resultant values in the faulty program. The rightmost column shows whether the original and faulty predicates give the same values.

To gain an idea of whether short-circuit rules can be useful for fault localization, we have run an initial experiment. We apply the whole test pool for the program from the Software-artifact Infrastructure Repository (SIR) [5], and record the counts of each of the five evaluation sequences for each test case. Following [11], we use the formula in Section 1 to calculate the evaluation biases for the set of successful test cases, and those for the set of failure-causing test cases. The results are shown as the histograms in Figure 2 . The distribution of evaluation biases over successful test cases and that over failure-causing test cases are given in pairs. The plots in Figures 2(a) to 2(e) are the respective distribution pairs of the five evaluation sequences. The plots in Figures 2(f) and 2(g) are those for the predicate-level, as used in previous work ([11]).

From the histograms in Figure 2, we observe that the distribution of evaluation biases for $e_{4}$ on successful test cases are drastically different from that of the failure-causing one. Indeed, it is the most different one among all pairs of histograms shown in the figure. We also observe from Table 1 that the fault in the code fragment can only be revealed when $e_{4}$ is used, because the fault does not affect the values in the other alternatives.

Our initial study indicates that it may be feasible to use evaluation sequences to identify a fault-relevant Statement more accurately. However, it is still uncertain how much the use of evaluation sequences will be beneficial to fault localization. We shall formulate our research questions in the next section and then investigate them experimentally in Section 4.

\section{Research Questions}

In this section, we shall discuss the research questions to be addressed by our controlled experimental study. We refer to a predicate-based statistical fault-localization technique as a base technique, and refer to the use of evaluation sequences in predicate execution counts as the fine-grained version of the base technique.

RQ1: In relation to the base technique, is the use of evaluation sequences for statistical fault localization effective?

RQ2: If the answer to RQ1 is true, is the effectiveness of using evaluation sequences significantly better than the base technique?

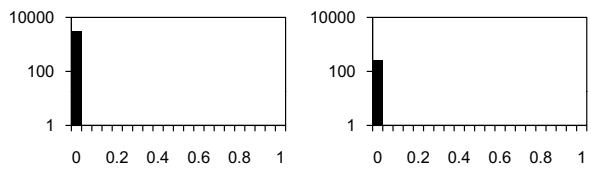

(a) $e s_{1}$ Plot

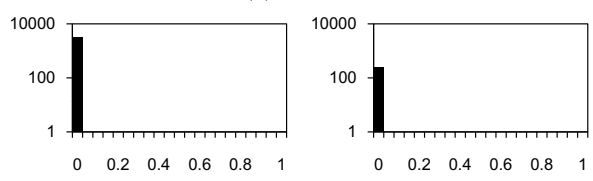

(b) $e s_{2}$ Plot

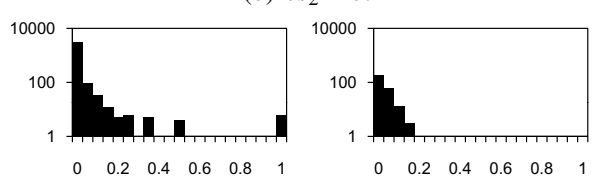

(c) $e s_{3}$ Plot
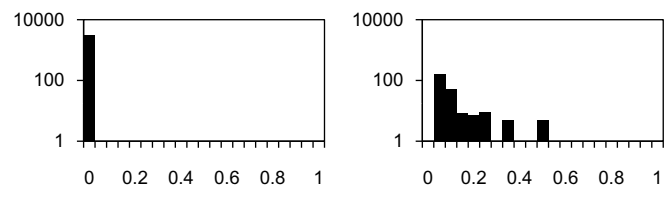

(d) $e s_{4}$ Plot

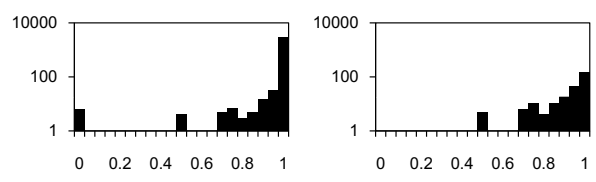

(e) $e s_{5}$ Plot
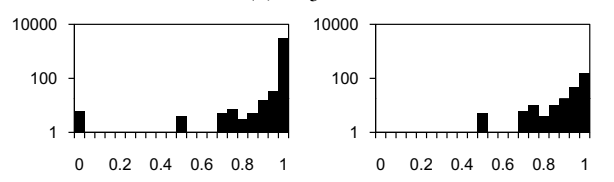

(f) predicate $_{\text {false }}$ Plot

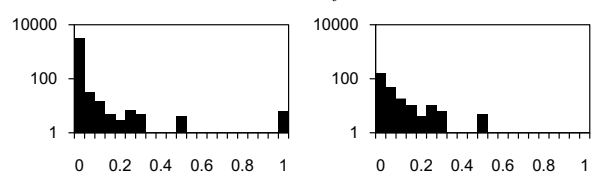

(g) predicate true Plot

Figure 2. Comparison of distributions of evaluation biases (x-axis: evaluation bias; y-axis: \# of test case).

RQ3: Do the execution statistics of different evaluation sequences of the same predicate differ significantly?

\subsection{Performance Evaluation}

Performance metrics are widely used to facilitate comparisons among different approaches. Renieris and Reiss [13] propose a ( $\mathrm{t}$-score) method of for measuring 
their fault-localization technique. The method is also adopted by Cleve and Zeller [3] and Liu et al. [11] to evaluate other fault-localization techniques.

Following [13], we also use t-scores to evaluate the fine-grained evaluation sequence approach in relation to the corresponding base techniques. We select two base techniques for study, namely SOBER [11] and CBI [10], because they are representative.

In brief, the t-score method takes a program $P$ and its marked faulty statements $S$ with a sequence of suspicious faulty statements $S^{\prime}$ as its input, and produces a value $V$ as output. The procedure to calculate the t-score is as follows: (i) Generate a Program Dependence Graph (PDG) $G$ for $P$. (ii) Using the dependence relations in the PDG as a measure of distance among statements, do a breadth-first search, which starts from some top or all statements in $S^{\prime}$, until reaching some statement in $S$. (iii) Return the percentage of searched statements (with respect to the total number of statements in $P$ ) as the value of $V$.

This measure is useful in assessing objectively the quality of proposed ranking lists of fault-relevant predicates and the performance of fault-localization techniques. Since the evaluation sequence approach is built on top of base techniques (such as SOBER and $\mathrm{CBI}$ ), we also use t-scores to compare different approaches in our controlled experiment to answer the research questions.

\subsection{Enabling Fine-Grained View of Base Techniques}

As we are interested in studying the impact of short-circuit evaluations and evaluation sequences for statistical fault localization, we need a method to incorporate the fine-grained view into a base technique. Intuitively, this provides execution statistics which may help statistical fault-localization techniques to identify the locations of faults more accurately.

We note that a base technique, such as SOBER or $\mathrm{CBI}$, conducts sampling of the predicates in a subject program to collect run-time execution statistics, ranks the fault relevance of the predicates. To assess the effectiveness of the selected set of predicates to locate faults, researchers may use t-scores to determine the percentage of code examined in order to discover the fault.

As such, given a set of predicates applicable to a base technique, we identify all legitimate evaluation sequences for each of these predicates. We then insert probes to the predicate location to collect the evaluation outcomes of individual conditions of these predicates. For each evaluation of the predicate, based on the evaluation outcomes of the individual conditions, we can determine the evaluation sequence which has been taken in the predicate evaluation. Hence, we collect the counts for individual evaluation sequences. By treating each evaluation sequence as a distinct (fine-grained) predicate in the base technique, the ranking approach in the base technique can be adopted to rank these finegrained predicates.

On the other hand, from the developers' viewpoint, it may be more convenient to recognize (through their eyeballs) the occurrence of an original predicate (than an evaluation sequence of the predicate) from the program text. Hence, it is to the benefit of developers to map the ranked evaluation sequences to their respective predicates and thus the corresponding statements.

Some measures should be taken in the above mapping procedure, however. Different evaluation sequences may receive different ranks. A simple mapping may thus result in a situation where a predicate occurs more than once in a ranking list. We choose to use the highest rank of all evaluation sequences for each individual predicate as the final rank of that predicate. This strategy also aligns with the basic idea of ranking predicates in SOBER and CBI. We refer to the finegrained approach as Debugging through Evaluation Sequences (DES).

\section{Controlled Experiment}

This section presents a controlled experiment and its results and analyses.

\subsection{Subject Programs and Test Cases}

In this study, we choose the Siemens suite of programs to conduct our experiment. They were originally created to support research on data-flow and control-flow test adequacy [7]. Our version of Siemens subject programs are obtained from the Software-artifact Infrastructure Repository (SIR) [5]. The Siemens suite consists of seven programs as shown in Table 2. A number of faulty versions are attached to each program. In our experiment, if any faulty version comes with no failure-causing cases, we do not include it in the experiment, since the base techniques ([10, 11]) require failure-causing test cases. We use a Unix tool, gcov, to collect the instrumentation log. 6 faulty versions that cannot be processed by gcov are excluded. As a result, we use 126 faulty versions in total.

Each of the Siemens programs is equipped with a test pool. According to the authors' original intention, the test pool simulates a representative subset of the input domain of the program so that test suites can be 
drawn from such test pools [5]. In the experiment, we follow the work of [11] to input the whole test pool to every technique to rank predicates or their evaluation sequences.

Table 2 shows the statistics of the test pools that we use in the experiment. The sources are obtained from SIR [5] (updated to January 10, 2008), including the average executable lines of code (EXE LOC), the total number of faulty versions (column (Faulty Ver.)), and the average percentage of compound statements with respect to all Boolean expression statements (column (B)). The column (A), which means the size of test pool, is from our fault matrix file. For instance, the print_tokens2 program has 10 faulty versions, which comprise 350-354 LOC, and its test pool contains 4115 test cases. On average, $5.4 \%$ of the Boolean expression statements of these faulty versions contain compound Boolean expressions. Other rows can be interpreted similarly.

From the column (B), we observe that in the subject programs, the percentages of predicate having more than one condition are low. This makes the research questions even more interesting to see whether such a low percentage would affect the performance of a base technique much.

\begin{tabular}{l||c|c|c|c|}
\hline Program & EXE LOC & Faulty Ver. & A & B \\
\hline \hline print_tokens & $341-342$ & 7 & 4130 & 1.7 \\
\hline print_tokens2 & $350-354$ & 10 & 4115 & 5.4 \\
\hline replace & $508-515$ & 32 & 5542 & 2.0 \\
\hline schedule & $291-294$ & 9 & 2650 & 3.2 \\
\hline schedule2 & $261-263$ & 10 & 2710 & 1.0 \\
\hline tcas & $133-137$ & 41 & 1608 & 2.4 \\
\hline tot_info & $272-274$ & 23 & 1052 & 5.6 \\
\hline
\end{tabular}

EXE LOC: executable lines of code.

Faulty Ver.: no. of faulty versions.

A: no. of test cases in the test pool.

B: the average percentage of compound Boolean

expressions to all Boolean expressions.

Table 2. Descriptive statistics of the subjects.

\subsection{Setup of Controlled Experiment}

In this section, we describe the setup of the controlled experiment. Using our tool, we produce a set of instrumented versions of these subject programs, including both the original and the faulty versions. Based on the instrumentation log as well as the coverage files created by gcov, we calculate the execution counts for the evaluation sequences, and finally rank the Boolean expression statements according to the description presented in Section 3. We also calculate how many faults have been successfully identified in the examined percentage of code at different t-scores (see Section 3).

The experiment is carried out on a DELL PowerEdge 1950 server with 2 4-core Xeon 5355 $(2.66 \mathrm{~Hz})$ processors, 8GB Physical Memory and 400GB Hard Disk equipped, serving a Solaris Unix with the kernel version of Generic_120012-14.

Our experimental platform is constructed using the tools of flex++ 2.5.31, bison++ 1.21.9-1, CC 5.8, bash 3.00.16(1)-release (i386-pc-solaris2.10), and sloccount 2.26 .

\subsection{Results and Analysis}

In this section, we present the experimental results, compare the relative effectiveness of the integrated approach with the base approach, and address the research questions one by one.

Answering RQ1: Is DES effective? Figures 3 and 4 show the results of SOBER against SOBER enabled with DES, and those of CBI against CBI enabled with DES, respectively. To ease our discussion, we refer to CBI enabled with DES as DES_CBI, and SOBER enabled with DES as DES_SOBER.

The $\mathrm{x}$-axis of each plot in these two figures shows the t-score. It represents the percentage of statements of the respective faulty program version affordable to be examined. The y-axis is the percentage of located faults with the given $t$-score. It is reported in [11] that "the top- $k$ result" means to use the best $k$ predicates in the ranked list to start applying the t-score method. We choose $k=5$ in the controlled experiment because, according to [11], the use of the best 5 predicates in the ranked list (top-5) will produce the best results for SOBER and CBI. We also show the top-5 plots for DES_SOBER and DES_CBI, which allow us to compare directly with the base techniques.

We observe from Figure 3 that DES_SOBER consistently achieves better average fault localization results (that is, more faults for the same percentage of examined code) than SOBER. For example, when checking $10 \%$ to $20 \%$ of the code, DES_SOBER can find at least 10 percent more faults than SOBER. As the percentage of examined code increases, however, the difference shrinks. This is understandable because, when an increasing amount of code has been examined, the difference between marginal increases of located faults will naturally be diminished. When all the faults are located or all the statements are examined, the two curves will attain the same percentage of located faults. We also observe from Figure 4 that DES_CBI also 


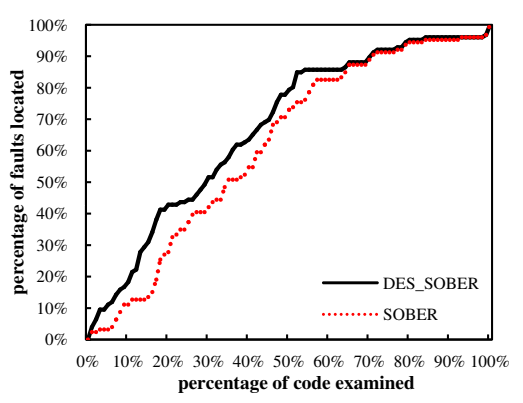

Figure 3. Direct comparison of DES_SOBER and SOBER.

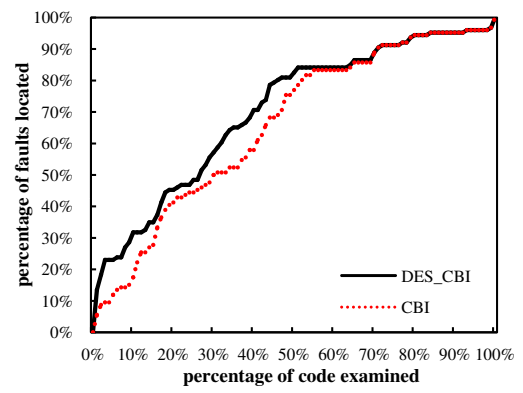

Figure 4. Direct comparison of DES_CBI and CBI.

outperforms CBI.

However, the visual differences between the curves seems to be small. To gain a more detailed picture, we further compare the two base techniques with their DES-enabled versions from another point of view. Figures 5 and 6 show the relative comparison of DES_SOBER and SOBER as well as DES_CBI and CBI on the Siemens suite of programs.

In Figure 5, the $\mathrm{x}$-axis of the plot is the percentage of code examined ( $t$-score); for a given percentage of code examined, the $\mathrm{y}$-axis shows a value that we call the relative percentage of faults located, calculated by the formula $\frac{\text { percentage of faults located by SOBER }}{\text { percentage of faults located by DES_SOBER }}-1$. Figure 6 can be interpreted similarly. In Figure 5 or Figure 6, the parts below the $\mathrm{x}$-axis indicates the relative percentage that the DES-enabled version of the respective technique (SOBER or CBI) outperforms the base version of the same technique. The portion above the $\mathrm{x}$-axis shows the opposite.

First, let us examine Figure 5. When the percentage of code examined is low, say $2 \%$ to $20 \%$, the curves for SOBER is far below the $\mathrm{x}$-axis. This shows that, when compared to DES_SOBER, SOBER locates less fault than DES_SOBER when the percentage of code examined is small. We can also find from Figure 6 that CBI locate less faults compared with DES_CBI. When the t-score increases, the difference shrinks as expected.

The results shows that on average, the evaluation sequence approach attains a relatively good fault-

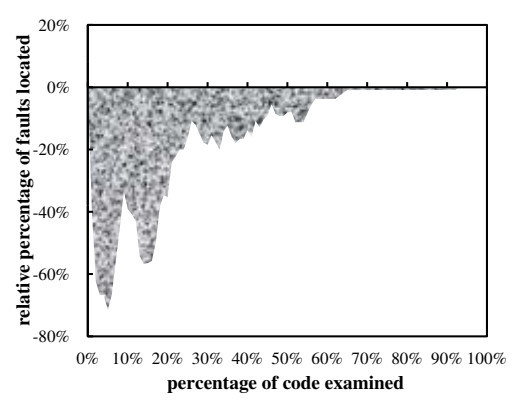

Figure 5. Relative comparison of DES_SOBER and SOBER.

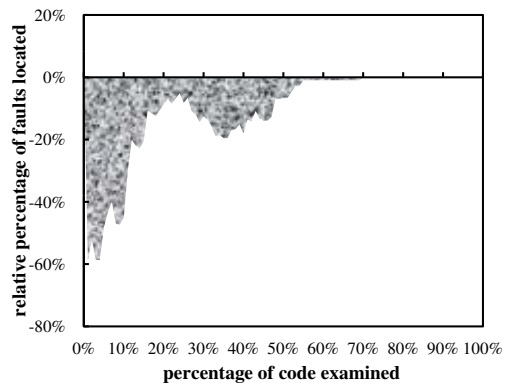

Figure 6. Relative comparison of DES_CBI and CBI.

localization effectiveness (as benchmarked with the base techniques). Therefore, we answer the first research question: the DES approach is effective.

Answering RQ2: Is DES better? In the above, we show that the DES approach is effective for fault localization. However, it is still unclear whether the difference between a base technique and its DESenabled version is not due to by chance. We further want to know: Does a technique enabled with the evaluation sequence approach differ significantly from the base technique? Is it better?

To answer these questions, we perform a U-test (Mann-Whitney U-test) to determine whether the DESenabled technique differ significantly from its base technique. The detailed procedure to analyze the data is as follows.

First, we subtract the percentage of located faults within the given t-score (values of $0 \%, 10 \%, \ldots, 100 \%$ ) of DES_CBI by that of CBI to obtain a set of sample data. Then we compare this set of sample data with another sample set of data containing only zeros to test the null hypothesis that DES_CBI and CBI are not significantly different. The result of the U-test for DES_CBI and CBI gives a p-value [4] less than 0.001, which successfully rejects the null hypothesis at 5\% significant level. It confirms that DES_CBI and CBI are significantly different. We also perform a U-test using the above procedure on DES_SOBER and SOBER 
instead of DES_CBI and CBI, respectively. The U-test gives a p-value less than 0.001 , which also successfully rejects the null hypothesis (at the 5\% significant level). From the above figures, we also observe that the DESenabled versions improve on their base techniques.

Our answer to RQ2 is: The DES-enabled techniques differ significantly from the respective base techniques. The answer to RQ2 also confirms that the short-circuit evaluation rules do have significant impacts on statistical fault localization.

Combining the answers to RQ1 and RQ2, the experimental results show that the DES approach has the potential to improve significantly the effectiveness of fault-localization techniques. ${ }^{2}$ They also show that short-circuiting is a significant factor in predicate-based statistical fault localization.

Answering RQ3: Do different evaluation sequences give the same result? To answer RQ3, we collect the execution statistics of all the evaluation sequences of the same Boolean expression to calculate the statistical differences between successful and failure-causing test cases. We perform a U-test between these evaluation biases for the set of evaluation sequences on the same predicate in successful and failure-causing test cases. The results of the U-test shows that $59.12 \%$ of the evaluation sequences have significant difference (at 5\% significant level) between the evaluation biases of the successful and failure-causing test cases. In the other words, $59.12 \%$ of the evaluation sequences are useful fault location indicators; whereas the other $40.87 \%$ evaluation sequences are not useful standalone fault predicators to differentiate failure-causing test cases from successful test cases.

The answer to RQ3 is that different evaluation sequences of the same predicate may have different potentials for fault localization. It will be interesting to analyze the results further to know the reasons.

\subsection{Threats to Validity}

We briefly summarize below the threats to validity in our controlled experiment.

Construct validity is related to the platform dependence issues when using the Siemens programs in SIR [5]. Since each program in SIR has a fault matrix file to specify the test verdict of every test case (i.e., whether a test case is successful or failure-causing) we create our own fault matrix file and carefully verify our test verdicts against the ones supplied with SIR. We observe that there are minor differences in test verdicts

\footnotetext{
${ }^{2}$ We are conservative about the conclusion because it is subject to external threats to validity to generalize the results.
}

between the two fault matrix files. We have carefully verified our setting, and believe that the difference is due to the platform dependence issues.

Internal validity is related to the risk of having confounding factors to affect the observed results. Following [11], in the experiment, each technique uses all applicable test cases to locate fault-relevant predicates in each technique. Using a test suite with different size may give a different result [11]. More evaluations on the impact of different test suites size on our technique will be welcoming. Another important factor is the correctness of our tool. Instead of adopting existing tools used in literature, we implement our own tools in $\mathrm{C}++$ to gain efficiency. Meanwhile, to reduce errors, we implemented and tested our tools carefully and adhere to the algorithms in literature. To align with previous work, we use the t-score method to compute the results of this experiment. The use of other metrics may produce different results.

External validity is the degree to which the results can be generalized to test real-world systems. We use the Siemens suite in the experiment to verify the research questions because they are commonly used by researchers in testing and debugging studies with a view to comparing different work more easily. Further applications of our approach to more medium to large size real-life programs would strengthen the external validity of our work. Each of the faulty versions in our subject programs contains one fault. Despite the competent programmer hypothesis, real-life programs may contain more than one fault. Though Liu et al. in [12] have demonstrated that such predicate-based techniques can be used to locate faults in programs that contain more than one fault, their effectiveness in this scenario is not well discussed. We shall address this threat in future work.

\section{Related Work}

There are rich categories of techniques in statistical fault localization. There are others besides the predicate-based category [10, 11].

Delta Debugging $[3,15]$ isolates failure-inducing input elements, produces cause-effect chains and locates the faults through analyzing the program state changes during the execution of a failed execution against a passed one.

Tarantula [9] ranks a statement according to its relevance to program faults, which is estimated by a ratio between the percentages of failure-causing and successful test cases that execute the statement. They further use Tarantula to explore ways of classifying test cases to enable multiple testing engineers to 
debug a faulty program in parallel [8]. Liblit et al. [10] propose a sparse sampling approach CBI to collect statistics of predicates for statistical fault localization. They further adapt CBI to exploit the execution statistics of compound Boolean expressions constructed from program predicates to facilitate statistical debugging [1].

Renieres and Reiss [13] find the execution traces difference between one failed execution and its "nearest neighbor" passed execution is effective for debugging. The statements in non-symmetric difference between a failed run and a passed run are regarded as faulty statements.

Baudry et al. [2] observe that some statements are always executed by the same set of test cases. They use a bacteriologic approach to remove test cases while maximizing the number of those dynamic basic blocks, and use the ranking algorithm in [9] to rank statements. They achieve using fewer test cases than Tarantula for the same fault-localization results.

Griesmayer et al. [6] use model checking to locate faults. By searching error traces, expressions that repair the original program are constructed.

\section{Conclusion}

Program debugging is time-consuming but important in software development. A major task in debugging is to locate faults. A common approach in statistical fault localization aims at locating program predicates that are close to faulty statements. This relaxes the requirement to pinpoint a fault location and has been shown empirically to be quite effective.

Following this popular trend, we would like to explore a better way to measure and rank predicates with respect to fault relevance. We observe that the fault-localization capabilities of various evaluation sequences of the same Boolean expression are not identical. Because of short-circuit evaluations of Boolean expressions in program execution, different evaluation sequences of a predicate may produce different resultant values. This inspires us to investigate the effectiveness of using Boolean expressions at the evaluation sequence level for statistical fault localization. Our experiment on the Siemens suite of programs shows that our approach is promising. Our future work will include locating faults in multi-fault programs using representative test suites.

\section{References}

[1] P. Arumuga Nainar, T. Chen, J. Rosin, and B. Liblit. Statistical debugging using compound Boolean predicates. In Proceedings of the 2007 ACM SIGSOFT International Symposium on Software Testing and Analysis (ISSTA 2007), pages 5-15. ACM Press, New York, NY, 2007.

[2] B. Baudry, F. Fleurey, and Y. Le Traon. Improving test suites for efficient fault localization. In Proceedings of the 28th International Conference on Software Engineering (ICSE 2006). ACM Press, New York, NY, 2006.

[3] H. Cleve and A. Zeller. Locating causes of program failures. In Proceedings of the 27th International Conference on Software Engineering (ICSE 2005), pages 342-351. ACM Press, New York, NY, 2005.

[4] G. E. Dallal. Historical background to the origins of p-values and the choice of 0.05 as the cut-off for significance. Available at http://www.tufts.edu/ gdallal/p05.htm.

[5] H. Do, S. G. Elbaum, and G. Rothermel. Supporting controlled experimentation with testing techniques: an infrastructure and its potential impact. Empirical Software Engineering, $10(4)$ : 405-435, 2005.

[6] A. Griesmayer, S. Staber, and R. Bloem. Automated Fault Localization for C Programs. Electron. Notes Theor. Comput. Sci., 174 (4): 157110661, 2007.

[7] M. Hutchins, H. Foster, T. Goradia, and T. Ostrand. Experiments on the effectiveness of dataflow- and controlflowbased test adequacy criteria. In Proceedings of the 16th International Conference on Software Engineering (ICSE '94), pages 191-200. IEEE Computer Society Press, Los Alamitos, CA, 1994.

[8] J.A. Jones, M. J. Harrold, and J.F. Bowring. Debugging in parallel. In Proceedings of the 2007 ACM SIGSOFT International Symposium on Software Testing and Analysis (ISSTA 2007), pages 16-26. ACM Press, New York, NY, 2007.

[9] J. A. Jones, M. J. Harrold, and J. Stasko. Visualization of test information to assist fault localization. In Proceedings of the 24th International Conference on Software Engineering (ICSE 2002), pages 467-477. ACM Press, New York, NY, 2002.

[10] B. Liblit, M. Naik, A. X. Zheng, A. Aiken, and M. I. Jordan. Scalable statistical bug isolation. in Proceedings of the 2005 ACM SIGPLAN Conference on Programming Language Design and Implementation (PLDI 2005), ACM SIGPLAN Notices, 40 (6): 15-26, 2005.

[11] C. Liu, L. Fei, X. Yan, S. P. Midkiff, and J. Han. Statistical debugging: a hypothesis testing-based approach. IEEE Transactions on Software Engineering, 32 (10): 831-848, 2006.

[12] C. Liu, X. Yan, L. Fei, J. Han, and S. P. Midkiff. SOBER: statistical model-based bug localization. In Proceedings of the Joint 10th European Software Engineering Conference and 13th ACM SIGSOFT International Symposium on Foundation of Software Engineering (ESEC 2005/FSE-13), ACM SIGSOFT Software Engineering Notes, 30 (5): 286-295, 2005.

[13] M. Renieres and S. P. Reiss. Fault localization with nearest neighbor queries. In Proceedings of the 18th IEEE International Conference on Automated Software Engineering (ASE 2003), pages 30-39. IEEE Computer Society Press, Los Alamitos, CA, 2003.

[14] I. Vessey. Expertise in debugging computer programs: a process analysis. International Journal of Man-Machine Studies, 23 (5): 459-494, 1985.

[15] A. Zeller and R. Hildebrandt. Simplifying and isolating failureinducing input. IEEE Transactions on Software Engineering, 28 (2): 183-200, 2002.

[16] A. X. Zheng, M. I. Jordan, B. Liblit, M. Naik, and A. Aiken. Statistical debugging: simultaneous identification of multiple bugs. In Proceedings of the 23rd International Conference on Machine Learning (ICML 2006), pages 1105-1112. ACM Press, New York, NY, 2006. 